\title{
ON THE ISOTROPIC GROUP OF A HOMOGENEOUS POLYNOMIAL
}

BY

\author{
SIU-MING HO( $\left.{ }^{1}\right)$
}

ABSTRACT. Let $G$ be the linear group leaving a homogeneous polynomial of degree $k$ fixed. The author shows that either the polynomial is a polynomial in fewer than the assigned number of variables or that the $(k-1)$ st prolongation of $G$ is 0 . The author also shows that this result is optimal.

Let $P^{k}\left[X_{1}, \cdots, X_{n}\right]$ be the space of homogeneous polynomials in $X_{1}, \cdots$, $X_{n}$ of degree $k$. Note that the linear group $G L(n)$ acts on $P^{k}\left[X_{1}, \cdots, X_{n}\right]$. If $A=\left(a_{i}^{j}\right) \in \mathrm{GL}(n)$ and $f\left(X_{1}, \cdots, X_{n}\right) \in P^{k}\left[X_{1}, \cdots, X_{n}\right]$, we denote the action of $A$ on $f$ by $A f$ where

$$
\text { Af }\left(X_{1}, \ldots, X_{n}\right)=f\left(\sum_{j=1}^{n} a_{1}^{j} X_{j}, \ldots, \sum_{j=1}^{n} a_{n}^{j} X_{j}\right)
$$

The Lie algebra $\mathrm{gl}(n)$ of $\mathrm{GL}(n)$ is the set of $n \times n$ matrices which also acts on $P^{k}\left[X_{1}, \cdots, X_{n}\right]$. If $a=\left(a_{i}^{j}\right) \in \mathrm{gl}(n), f \in P^{k}\left[X_{1}, \cdots, X_{n}\right]$ then

$$
a \cdot f\left(X_{1}, \ldots, x_{n}\right)=\sum_{i, j}^{n} \frac{\partial f}{\partial X_{i}} a_{i}^{j} X_{j}
$$

This is obtained by writing $A=\exp (t a)$ and differentiating (1) with respect to $t$.

Let $G$ be the Lie sugbroup of $G L(n)$ which leaves $f \in P^{k}\left[X_{1}, \cdots, X_{n}\right]$ invariant, and $g$ the Lie algebra of $G$. By formula (2) we have the following.

Lemma. The Lie algebra $g$ of $G$ consists of those matrices $\left(a_{i}^{j}\right)$ for which $\Sigma_{i, j}^{n} \partial f / \partial X_{i} a_{i}^{j} X_{j}=0$.

For each element $a=\left(a_{i}^{j}\right) \in \mathrm{gl}(n)$ we can think of $\left(A^{1}, \ldots, A^{n}\right)$ as an $n-$ tuple of homogeneous polynomials of first order, where $A^{i}=\sum_{j=1}^{n} a_{i}^{j} X_{j}$. From now on we identify $\mathrm{gl}(n)$ with the space of $n$-tuple of homogeneous polynomials of first order. So we can also think of the subspace $g$ of $\mathrm{gl}(n)$ as a subspace of the above space.

Received by the editors November 3, 1972.

AMS (MOS) subject classifications (1970). Primary 53C10.

Key words and phrases. $G$-structure, prolongation.

$\left({ }^{1}\right)$ This research was partially supported by a grant from NSF, P22927X1. 
For any Lie subalgebra $g$ (see [3]) of $\mathrm{gl}(n)$ we define the first prolongation $g^{(1)}$ of $g$ as the set of all $n$-tuples. $\left(A^{1}(X), \cdots, A^{n}(X)\right)$, where $A^{i}$ is a homogeneous polynomial of degree two in $X_{1}, \cdots, X_{n}$ and all the first derivatives, $i=1, \cdots, n,\left(\partial A^{1} / \partial X_{i}, \cdots, \partial A^{n} / \partial X_{i}\right)$ lie in $g$. Similarly we define the $k$ th prolongation $g^{(k)}$ of $g$ as the set of all $n$-tuples $\left(A^{1}(X), \cdots, A^{n}(X)\right)$ where $A^{i}(X)$ is a homogeneous polynomial of degree $k+1$ in $X_{1}, \cdots, X_{n}$, and all the $k$ th derivatives $\left(\partial^{k} A^{1} / \partial X^{\alpha}, \ldots, \partial^{k} A^{n} / \partial X^{a}\right),|\alpha|=k$, lie in $g$.

Definition. The algebra $g$ is of finite type if $g^{(k)}=0$ for some $k$ (and, therefore for all large $k) G$ is said to be of type $k$ if $g^{(k-1)} \neq 0$ and $g^{(k)}=0$.

Definition. $f(X)$ is a nonsingular homogeneous polynomial if $f$ cannot be written as a polynomial in fewer than $n$ variables.

We have the following result:

Theorem. If $f \in P^{k}\left[X_{1}, \cdots, X_{n}\right]$ and nonsingular then $g^{(k-1)}=0$.

Proof. If $\left(A^{1}(X), \cdots, A^{n}(X)\right) \in G^{(k-1)}$, by definition

$$
\left(\partial^{k-1} A^{1} / \partial X^{\alpha}, \ldots, \partial^{k-1} A^{n} / \partial X^{\alpha}\right), \quad|\alpha|=k-1, \epsilon g .
$$

then

$$
\sum_{i=1} \frac{\partial f}{\partial X_{i}} \frac{\partial^{k-1}}{\partial X^{a}} A^{i}=0 \text { for all }|a|=k-1
$$

By repeated application of Euler's ident ity for homogeneous functions we can rewrite this as

$$
\sum_{i=1}^{n} \frac{\partial^{k-1}}{\partial X^{a}}\left(\frac{\partial f}{\partial X_{i}}\right) A^{i}=0 \text { for all }|\alpha|=k-1 .
$$

However $\partial^{k-1} / \partial X^{a}\left(\partial f / \partial X_{i}\right)$ are constants, so choosing any $X_{0}$ such that the $A^{i}\left(X_{0}\right)$ are not all zero, and setting $b_{i}=A^{i}\left(X_{0}\right)$ we get

$$
\sum \frac{\partial^{k-1}}{\partial X^{a}}\left(\frac{\partial f}{\partial X_{i}}\right) b_{i}=0 \text { for all }|a|=k-1
$$

This implies $\Sigma b_{i} \partial f / \partial X_{i}=0$, in other words $f$ can be written as a polynomial in $n-1$ variables which contradicts the hypotheses. Q.E.D.

In what follows we construct some examples which show that the above theorem is the best possible result we can get. Let 


$$
f\left(x_{1}, x_{2}, x_{3}, x_{4}, x_{5}\right)=\left|\begin{array}{lll}
x_{3} & x_{4} & x_{5} \\
x_{1} & x_{2} & 0 \\
0 & x_{1} & x_{2}
\end{array}\right|=x_{3} x_{2}^{2}-x_{4} x_{1} x_{2}+x_{5} x_{1}^{2} \text {. }
$$

It is easily seen that $f$ cannot be written as a polynomial fewer that 5 variables. Let $\left(A^{1}, A^{2}, A^{3}, A^{4}, A^{5}\right)$ be a 5-tuple of homogeneous polynomials of second degree where $A^{1}=0, A^{2}=0, A^{3}=X_{1}^{2}, A^{4}=2 X_{1} X_{2}, A^{5}=X_{2}^{2}$. Then

$$
\sum_{i=1}^{n} \frac{\partial f}{\partial X_{i}} \frac{\partial A^{i}}{\partial X_{j}}=0 \text { for all } j=1,2,3,4,5 \text {. }
$$

This implies $\left(A^{1}, A^{2}, A^{3}, A^{4}, A^{5}\right) \in g^{(1)}$, so $g^{(1)}$ is nontrivial.

Let $f \in P^{k-2}\left[X_{1}, X_{2}, \cdots, X_{k}\right]$ be defined as follows:

$$
f\left(X_{1}, X_{2}, \ldots, X_{k}\right)=\left|\begin{array}{llll}
A^{1} & =0, & A^{4}=X_{1}^{k-4} X_{2}, \\
A^{2}=0, & A^{i}=X_{1}^{k-i} X_{2}^{i-3}, \\
A^{3}=X_{1}^{k-3}, & A^{k}=X_{2}^{k-3} ; \\
\frac{\partial^{k-4} A^{3}}{\partial X_{1}^{k-4}} & \frac{\partial^{k-4} A^{4}}{\partial A_{1}^{k-4}} & \cdots & \frac{\partial^{k-4} A^{k}}{\partial X_{1}^{k-4}} \\
\frac{\partial_{1}^{k-5} \partial X_{2}}{\partial X_{2}^{k-4}} & \frac{\partial^{k-4} A^{4}}{\partial X_{1}^{k-5} \partial X_{2}} & \cdots & \frac{\partial^{k-4} A^{k}}{\partial X_{1}^{k-5} \partial X_{2}} \\
\frac{\partial^{k-4} A^{4}}{\partial X_{2}^{k-4}} & \cdots & \frac{\partial^{k-4} A^{k}}{\partial X_{2}^{k-4}}
\end{array}\right| .
$$

It is easily seen that $f$ is nonsingular and also

$$
\sum_{i=1}^{n} \frac{\partial f}{\partial X_{i}} \frac{\partial^{k-4} A^{i}}{\partial X^{a}}=0 \text { for all }|a|=k-4
$$

so we have $g^{k-4}$ of $f$ is nontrivial.

Note that the first example of $g^{(1)} \neq 0$ occurs in dimension 5 . Using a result of Gordan-Noether (see [1]) one can show that, for dimensions $\leq 4, g^{(1)}=0$ and, for dimension $5, g^{(2)}=0$.

The result above has interesting consequences for the problem of when a partial differential operator can be transformed to a constant coefficient operator see [2]. 


\section{BIBLIOGRAPHY}

1. P. Gordan and M. Noether, Ueber die algebraischen Formen deren Hesse'che Determinante identisch verschwindet, Math. Ann. 10 (1876), 547-568.

2. V. Guillemin and I. M. Singer, Differential equations and G-structures, U. S.-Japan Seminar in Differential Geometry (Kyoto, 1965), Nippon Hyoronsha, Tokyo, 1966, pp. 34-36. MR 35 \#4840.

3. I. M. Singer and S. Stemberg, On the infinite groups of Lie and Cartan. I, The transitive groups, J. Analyse Math. 15 (1965), 1-114. MR 36 \#911.

DEPARTMENT OF MATHEMATICS, MASSACHUSETTS INSTITUTE OF TECHNOLOGY, CAMBRIDGE, MASSACHUSETTS 02139 